Original research article

\title{
Improved timeliness for reporting of acute diarrhoeal disease under surveillance overtime: Evaluation of integrated disease surveillance programme in North 24 Parganas, West Bengal, India, 2015
}

\author{
Falguni Debnath ${ }^{\mathrm{a}, \mathrm{b}, *}$, Manickam Ponnaiah ${ }^{\mathrm{b}}$ \\ a ICMR -National Institute of Cholera and Enteric Diseases, Kolkata, India \\ ${ }^{\mathrm{b}}$ ICMR -National Institute of Epidemiology, Chennai, India
}

\section{A R T I C L E I N F O}

\section{Article history:}

Received 9 August 2017

Received in revised form 16 October 2017

Accepted 18 October 2017

Available online 18 October 2017

\section{Keywords:}

Diarrhoeal disease

Disease surveillance

Diarrhoeal disease outbreaks

Timeliness of reporting

\begin{abstract}
A B S T R A C T
Introduction: India's Integrated Disease Surveillance Programme (IDSP) in North 24 Parganas District of West Bengal reported outbreaks of acute diarrhoeal diseases (ADD) during 2010-2014 without laboratory confirmation. Hence, we described the surveillance system and evaluated in terms of selected surveillance attributes: simplicity, acceptability, timeliness of reporting of ADD/outbreaks, representativeness, positive predictive value, usefulness.

Methods: We described the surveillance through review of documents, manuals, records and interview of surveillance staff. For evaluation of the surveillance system, cross sectional study was conducted among 95 sampled Auxiliary Nurse Midwives (ANMs), 22 Block Medical Officers (BMOHs) of the District and also abstracted data from records. We developed indicators for surveillance attributes and computed proportions for the same. We computed descriptive statistics for timeliness of reporting.

Results: Seventeen percent $(n=15)$ of first ANMs and six of nineteen medical officers had correct knowledge on ADD case definition. One-fourth of the ANMs reported lack of communication, training, supervision and lack of feedback in preceding six months. Median time taken to intimate district rapid response team (RRT) and to collect specimen from the date of first case reporting was the same day of reporting of index case (Range: 0-2 days vs. 0-3 days) during ADD outbreaks in 2014. Of the five reported ADD outbreaks in 2014, one was laboratory confirmed.

Conclusions: Knowledge regarding case definition was poor at all level of health workers. Existing supervision and feedback mechanism needs to be improved. Timeliness of reporting to district RRT and specimen collection during outbreak had improved overtime.
\end{abstract}

(c) 2017 Published by Elsevier, a division of RELX India, Pvt. Ltd on behalf of INDIACLEN.

\section{Introduction}

Communicable disease control is an important public health priority at international level to stop spread of different contagious diseases. ${ }^{1}$ In order to make policy for effective control of communicable diseases, every country requires effective and efficient surveillance and reporting system at all levels. ${ }^{2}$ With this demand in background integrated disease surveillance was introduced in many countries to sum up all the surveillance activities, which finally add up to the national surveillance system. ${ }^{2}$ The core activities of surveillance system are - case

\footnotetext{
* Corresponding author at: Division of Epidemiology, ICMR - National Institute of Cholera and Enteric Diseases, P- 33, CIT Road, Scheme - XM, Beliaghata, Kolkata, West Bengal, 700010, India.

E-mail addresses: falgunidebnath@yahoo.in (F. Debnath), manickamp@gmail.com (M. Ponnaiah).
}

detection, reporting, investigation, confirmation, analysis, interpretation, action and support functions. ${ }^{2}$ As Acute diarrhoeal disease (ADD) and pneumonia are major killer diseases in underfive age group globally, ${ }^{3}$ they are under routine surveillance in many countries.

In India, Integrated disease surveillance programme (IDSP) was launched in 2004 with the objective of strengthening the disease surveillance in the country by establishing a decentralized state bases surveillance system for epidemic prone diseases to detect the early warning signals, so that timely and effective public health actions can be initiated in response to health challenges in the country at district, state, national level. ${ }^{4}$ In India, ADD contributes $13 \%$ to all deaths in children under 5 years of age and an estimated 300,000 children die each year. ${ }^{5}$

IDSP was launched in West Bengal in the year 2007. Our analysis of ADD district surveillance data from North 24 Parganas district indicated that outbreaks of ADD increased from 4 in 2010 to 14 in 
2013. ${ }^{6}$ However, few of the outbreaks were laboratory confirmed. Information on reasons for absence of such confirmation are not available with us. In this context, we described the ADD surveillance and evaluated the same for surveillance attributes in North 24 Parganas district. ${ }^{7}$

\section{Methods}

\subsection{Describing the acute diarrhoeal surveillance under IDSP}

To describe the acute diarrhoeal surveillance under IDSP, IDSP guidelines, manuals and records were reviewed. District epidemiologist, IDSP data manager, first level Auxiliary Nurse Midwives (first ANMs) and Block Medical Officers of Health (BMOH) of the district were interviewed by principal investigator using semi structured questionnaire.

\subsection{Evaluation of acute diarrhoeal surveillance under IDSP}

We evaluated the surveillance system in-terms of selected surveillance attributes like simplicity, acceptability, representativeness, timeliness of regular reporting/reporting during ADD outbreaks, positive predictive value and usefulness. It was evaluated for a reference period of January to December'2014, but specifically the timeliness of reporting during outbreaks was evaluated for 2010 to 2014. We used data abstraction form to collect data from the IDSP records.

Ninety-five of total 738 first ANMs of the district were needed to be interviewed assuming that $50 \%$ of them were having correct knowledge about acute diarrhoeal disease case definition, $10 \%$ of precision, $95 \%$ of confidence interval and accounting for $10 \%$ of nonresponse. Sample size was calculated using Epi Info Version $3 .^{8}$ Random number list was generated by investigators using Epi Info to select the first ANMs for interview. Apart from that we also proposed to interview all the $22 \mathrm{BMOHs}$.

\subsection{Operational definitions}

Simplicity: Refers to the structure of the surveillance system and its' operational ease. $^{7}$

Acceptability: Refers to the willingness of the institutions to participate in the surveillance system. ${ }^{7}$

Representativeness: Refers to the ability of the system to describe the health events accurately in terms of time, place, person. $^{7}$

Timeliness of regular $A D D$ reporting/outbreak reporting: Refers to the ability of the system to trigger action in time. ${ }^{7}$

Positive predictive value: Refers to the probability that the cases captured by the system is a true case. ${ }^{7}$

Usefulness: Refers to the relevance of the system in terms of feeding information for action. ${ }^{7}$

Acute diarrhoeal disease was defined as passage of three or more loose stool or watery stools in past 24 hours with or without dehydration. $^{9}$

Trigger event for acute diarrhoeal disease was defined as a single case of cholera or epidemiologically linked case of diarrhea/a case of severe dehydration or death due to diarrhea/clustering of cases particularly village or ward where $>10$ houses having at least one case of loose stools irrespective of age. ${ }^{9}$

Time taken to report to district rapid response team about the occurrence of the outbreak was calculated from the date of reporting of first case (index case).

Time taken to specimen collection during acute diarrhoeal outbreak was calculated from the date of reporting of first case (index case).

Table 1

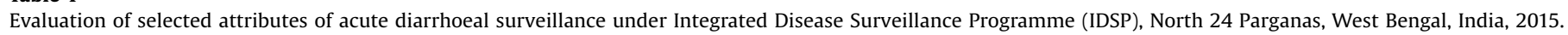

\begin{tabular}{|c|c|c|c|c|}
\hline Attribute & Indicator used to evaluate & $\mathrm{N}$ & $\mathrm{n}$ & $\%$ \\
\hline \multirow[t]{6}{*}{ Simplicity } & \multirow[t]{2}{*}{ Flow of information in this surveillance system is simple } & $\mathrm{BMOH}=19$ & 15 & 79 \\
\hline & & first $\mathrm{ANM}=88$ & 66 & 75 \\
\hline & \multirow[t]{2}{*}{ Form is simple to fill } & $\mathrm{BMOH}=19$ & 17 & 89 \\
\hline & & first $\mathrm{ANM}=88$ & 65 & 74 \\
\hline & \multirow[t]{2}{*}{ Form is not time consuming } & $\mathrm{BMOH}=19$ & 14 & 74 \\
\hline & & first $\mathrm{ANM}=88$ & 76 & 86 \\
\hline \multirow[t]{2}{*}{ Acceptability } & Submission of weekly reports for 52 weeks by health care facilities & 827 & 824 & 99.6 \\
\hline & Submission of nil reporting throughout 52 weeks in a year & 824 & 133 (132 HSCs,1PHC) & 16 \\
\hline \multirow[t]{3}{*}{ Representativeness } & Submission of reports by Public healthcare facilities (weekly) & 825 & 822 & 99.6 \\
\hline & Submission of reports by Private healthcare facilities (weekly) & 238 & 2 & 0.01 \\
\hline & Submission of reports by Municipalities in one year & 27 & 0 & 0 \\
\hline \multirow[t]{6}{*}{ Positive predictive value } & \multirow[t]{2}{*}{ Correct knowledge about ADD case definition } & $19 \mathrm{BMOHs}$ & 6 & 31 \\
\hline & & 88 first ANMs & 15 & 17 \\
\hline & \multirow[t]{2}{*}{ Correct knowledge about trigger event for ADD } & $19 \mathrm{BMOHs}$ & 11 & 58 \\
\hline & & 88 first ANMs & 7 & 8 \\
\hline & \multirow{2}{*}{ Trained in surveillance system } & $19 \mathrm{BMOHs}$ & 7 & 37 \\
\hline & & 88 first ANMs & 70 & 80 \\
\hline \multirow[t]{2}{*}{ Positive predictive value } & \multirow[t]{2}{*}{ Received refresher training in past six months } & $19 \mathrm{BMOHs}$ & 0 & 0 \\
\hline & & 88 first ANMs & 4 & 5 \\
\hline \multirow[t]{5}{*}{ Usefulness } & No. of laboratory confirmed cholera outbreaks 2014 & - & 1 & - \\
\hline & No. of ADD outbreaks in which specimens (stool, water) sent for laboratory confirmation & 5 & 5 & 100 \\
\hline & No. of etiologically confirmed ADD outbreak & 5 & 1 & 20 \\
\hline & No. of ADD outbreaks informed to district RRT in $<48$ hours of first case reporting & 5 & 4 & 80 \\
\hline & No. of ADD outbreak investigated within 48 hours of first case reporting by district RRT & 5 & 4 & 80 \\
\hline
\end{tabular}




\subsection{Data analysis}

Proportions for surveillance attributes - simplicity, acceptability, representativeness, timeliness of regular reporting, positive predictive value and usefulness were computed. Regarding timeliness of reporting during outbreaks, we calculated descriptive statistics (Median, IQR) for time taken from identification of index case to intimate the district rapid response team about occurrence of the outbreak and time taken for collection of specimen. Boxplots were drawn for the same.

\subsection{Human subject protection}

Approval from Institutional human ethics committee of National Institute of Epidemiology, Chennai, India was obtained. We obtained permission from the District chief health official before initiating the study. Written informed consent in local language from the study participants was obtained before administering questionnaire. We used unique identity numbers in the study tools and maintained confidentiality.

\section{Results}

\subsection{Description of acute diarrhoeal surveillance under IDSP}

ADD is one of the 26 diseases under surveillance in IDSP in the study district (Population $=10$ million). This surveillance system was established with an objective of early detection and response to disease outbreaks. Collection of data, compilation of data, analysis and interpretation, follow up action, providing feedback in the form of IDSP alerts are the different components of the surveillance activity. Three types of reporting formats are used for regular reporting. Form "S (Syndromic)" is filled by the first ANMs at health sub center (HSC) level. Form "P (Probable)" is filled at primary health center ( $\mathrm{PHC}$ )/block primary health center (BPHC) level by the doctors. Form "L (Laboratory)" comes from the laboratories. This is a weekly reporting system. All the forms (S, P,L) reach to the district surveillance unit weekly and thereafter, district data manager uploads the reports in the IDSP portal making a copy to the state surveillance unit. Different reporting format is used during disease outbreaks as and when required. Thirteen District Hospitals/Sub divisional hospitals/State general hospitals, 22 BPHCs, 50 PHCs, 742 HSCs and only two private laboratories have taken part in this surveillance system. The weekly data is only analyzed at the district surveillance unit. According to the guideline of this surveillance system, laboratory test is only required during acute diarrhoeal outbreaks. For quick response and control of outbreaks, block medical officer and his team informs the district rapid response team as early as possible about the occurrence of outbreak and takes control measures, following which district rapid response team investigates the outbreak along with the block team.

\subsection{Evaluation of acute diarrhoeal surveillance under IDSP in terms of selected attributes}

Nineteen Block Medical Officers of Health and 88 first ANMs of the district were interviewed. According to $79 \%$ of the block medical officers (BMOH) $(n=15), 75 \%$ of the first ANMs $(n=66)$, the flow of information in this surveillance system was simple. However, $24 \%$ of the first ANMs told about lack of communication, training \& supervision and $23 \%$ of them told that they need space in the form to write about the episodes of frequent mucoid stools in 24 hours (Table 1 ).
Out of the 824 health care facilities who reported weekly for 52 weeks, 16\% (132 HSCs, 1 PHC) had done nil reporting throughout the year.

All 824 facilities who reported for 52 weeks, had reported within one week after the last date of every reporting week at least for 40 weeks in that year (Table 1$).^{10}$

In between 2010 to 2014,28 acute diarrhoeal outbreaks were reported. In 2010, the median time taken to report to district rapid response team from the date of first case reporting was 2.5 days (IQR, 2 to 5 days) (Fig. 1). Whereas, in 2014, it was zero day (IQR, 0 to 2 days).

In 2010, the median time taken for specimen collection from the date of first case reporting was 3 days (IQR, 2 to 6 days) (Fig. 2). Whereas, in 2014, it was zero day (IQR, 0 to 3 days).

Among the interviewed, $31 \%$ of the BMOHs $(n=6)$ and $17 \%$ of the first ANMs $(n=15)$ knew the case definition of acute diarrhoeal disease laid down by IDSP (Table 1$) .58 \%$ of the BMOHs $(n=11)$ and $8 \%$ of the first ANMs $(n=7)$ knew the trigger event for acute diarrhoeal disease.

\section{Discussion}

We described and evaluated ADD surveillance under IDSP in North 24 Parganas district of West Bengal. The system and the forms were considered simple. Two private health care facilities and none of the 27 municipalities participated. One-sixth of the participating facilities did not report any cases. Timeliness of reporting during ADD outbreaks improved from 2010 to 2014, however, only one of the five acute diarrhoeal outbreak was laboratory confirmed in 2014. Correct knowledge about ADD definition was poor among medical officers and peripheral health workers and they reported about the lack of training and feedback in the system.

Poor participation of the private healthcare facilities and no participation from municipalities are a matter of concern to the

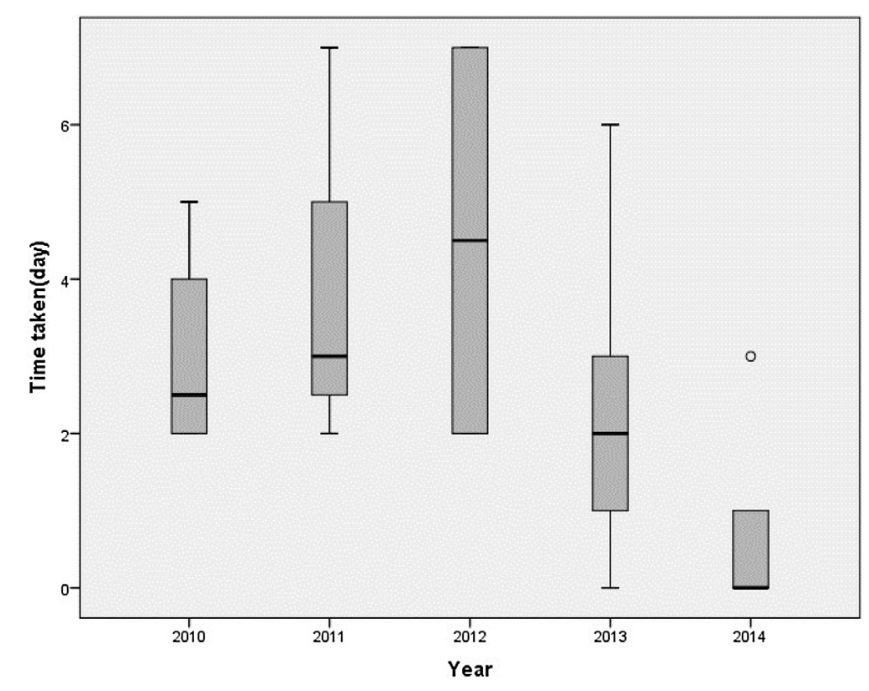

Fig. 1. Time taken to report during the acute diarrhoeal disease outbreaks to district Rapid Response Team from the date of first case reporting, North 24 Parganas, West Bengal, India, 2010-14.

description: In this boxplot, $\mathrm{X}$ axis showing the years whereas $\mathrm{Y}$ axis showing the time taken in days to intimate the district rapid response team from the date of first case reporting. In 2010, the median time taken to report to district rapid response team from 1 st case reporting was 2.5 days (IQR, 2 to 5 days), in 2011, it was 3 days (IQR, 2 to 7days), in 2012, it was 4.5 days (only 2 outbreaks were reported), in 2013, it was 2 days (IQR, 1 to 3 days) and in 2014, it was 0 day (IQR, 0 to 2 days). orepresents outlier. 


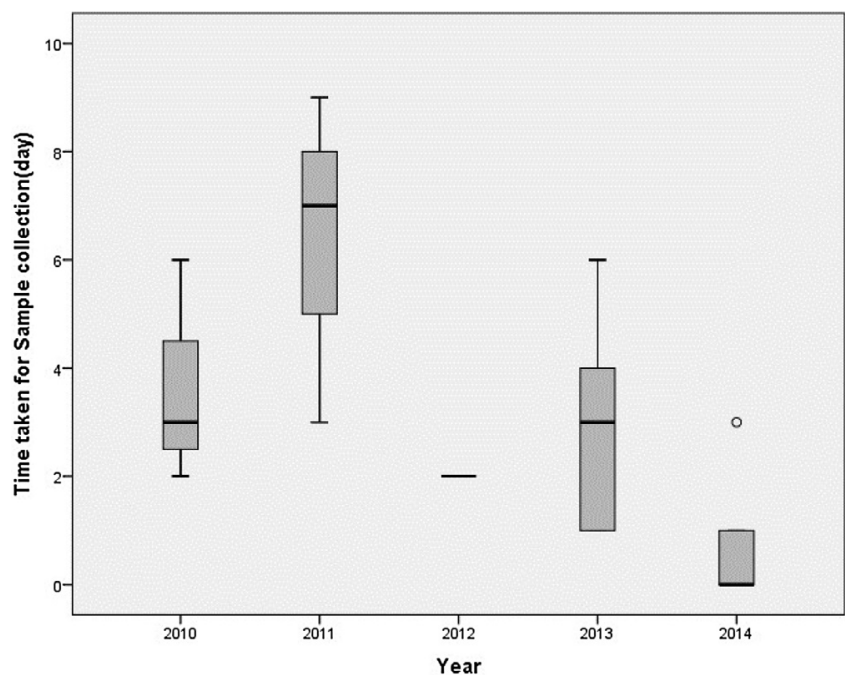

Fig. 2. Time taken for specimen (stool) collection from the date first case reporting during acute diarrhoeal disease outbreaks, North 24 Parganas, West Bengal, India, 2010-14.

description: In this boxplot, $\mathrm{X}$ axis showing the years whereas $\mathrm{Y}$ axis showing the time taken in days to collect specimen (stool) from the date of first case reporting. In 2010 , the median time taken to collect specimen from 1 st case reporting was 3 days (IQR, 2 to 6 days), in 2011, it was7 days (IQR, 3 to 9days), in 2012, it was 2 days (only in 1 outbreak, specimens were sent for laboratory confirmation), in 2013, it was 3 days (IQR, 1 to 5 days) and in 2014, it was 0 day (IQR, 0 to 3 days). o-represents outlier.

district health officials. North 24 Parganas is the highest populous district in India. More participation would ensure capturing of the actual disease burden. Though strong public private partnership has been identified as one of the four important elements of a frontline surveillance system, ${ }^{2}$ but so far, the district is unable to achieve that.

Nil reporting from one-sixth of the health facilities throughout the year raises concerns about existing supervision mechanism within IDSP. Moreover, one fourth of the interviewed first ANMs mentioned lack of communication and supervision in this existing system. Regular supervision and support from supervisor are globally identified important factors for good performance among all level of health care workers. ${ }^{11,12}$ One of the important goals of a functional disease surveillance and notification system is to detect and monitor diseases, other events with potential threat to the health of the public in order to provide rationale for public health action. ${ }^{13}$ Moreover, nil/no reporting creates a chance for public health authorities to miss out opportunities to initiate control measures in some of acute diarrhoeal outbreaks at the earliest.

The control of an outbreak depends on early detection followed by rapid structured investigation and confirmation. ${ }^{13-15}$ Timeliness of reporting to district rapid response team during acute diarrhoeal outbreaks and timeliness of specimen collection during outbreaks had improved over time though all the outbreaks were not laboratory confirmed. Timeliness of regular reporting was satisfactory.

For the surveillance system to be functional, appropriate, timely and responsive, effective functioning of the health care workers at regional/state/national level is most important and thus human factor is more important than design of the surveillance system. ${ }^{2}$ Hence provision of periodic in-service training, support, feedback, motivation need to be guaranteed within the design of the surveillance system. In our study we found that knowledge regarding acute diarrhoeal case definition and trigger event was dissatisfactory at all level of health care workers, though majority of them finds this surveillance simple. Moreover, less than one fourth of the interviewed first ANMs received any feedback regarding their activities in preceding six months. All these indicate towards the lack of timely refresher trainings, active supervision - monitoring, active feedback mechanism within the system. Since at the peripheral level, surveillance activities are done in addition to the huge clinical workload, it is critical that the health care workers involved in surveillance activities can regularly see the impact of their work as this will maintain their momentum to work effectively in the surveillance system. Hence, having an inbuilt feedback loop in the surveillance system is necessary.

Our study had few limitations, first, evaluation of the acute diarrhoeal surveillance under IDSP was done in terms of selected attributes. Second, due to logistic constraints interviewing all the block medical officers of the district was not possible. Third, the questionnaires used in this study may not be the universal one for measuring knowledge of the health care workers or collecting information on different factors. But we preferred to develop the tool locally as no standard tool was available. Moreover, knowledge level was measured only for diarrhoeal case definition and its trigger event as per IDSP guidelines. So, chance of underestimating their knowledge was absent in our study.

Ongoing acute diarrhoeal surveillance under IDSP is simple. Timeliness of regular reporting was satisfactory. Timeliness of reporting to district rapid response team and specimen collection during acute diarrhoeal outbreaks had improved overtime but all the acute diarrhoeal outbreaks were not laboratory confirmed. Poor public private partnership in the existing acute diarrhoeal surveillance under IDSP is a matter of concern. Knowledge regarding diarrhoeal case definition and its trigger event was poor at all level of health care workers. Supervision and feedback mechanism was poor in this existing acute diarrhoeal surveillance under IDSP in North 24 Parganas district of West Bengal. Based on our study findings, we recommend planning for periodical (at least six monthly) in service refresher trainings for the health workers specifically focusing on IDSP operations including case definitions and laboratory aspects. Improvement of the supervision and feedback mechanism in this existing surveillance system is suggested.

\section{Funding}

This study was partially funded by ICMR - National Institute of Epidemiology, Chennai, India.

\section{Conflicts of interest}

None declared.

\section{Contribution of the authors}

Dr. Falguni Debnath has contributed in terms of conceiving the idea of this study, designing, data collection, analysis, writing first draft of the manuscript. Dr. Manickam Ponnaiah has contributed in terms of designing this study, analysis, reviewing and editing the manuscript.

\section{References}

1. Janati A, Hosseiny M, Gouya MM. Communicable Disease Reporting Systems in the World: A Systematic Review Article. Iran J. Public Health. 2015;44 (11):1453-1465.

2. States M, Strate W. Group A et al. An integrated approach to communicable disease surveillance. Weekly Epidemiol. Rec. 2011;47:521-540.

3. Children: Reducing mortality. Fact Sheet. Media Center, World Health Organization; 2016 Available from: http://www.who.int/mediacentre/ factsheets/fs178/en/ (Accessed 25 May 2017). 
4. Lal A, Tandon R, Upadhaya S. Joint Implementation Review. India: Integrated Disease Surveillance Project; 2009 Available from: http://www.idsp.nic.in/ (Accessed 5 August 2016).

5. Bassani DG, Kumar R, Awasthi S, et al. Causes of neonatal and child mortality in India: a nationally representative mortality survey. Lancet. 2010;376:18531860.

6. Debnath F. Descriptive epidemiology of acute diarrhoeal disease and outbreaks in North 24 Parganas district, West Bengal, 2010-2014. The Child AND Newborn. J. West Bengal Acad. Pediatrics. 2016;20:6-9.

7. Klaucke DN, Buehle JW, Thacker SB, Guidelines for Evaluating Surveillance Systems MMWR. 1988;vol. 37::1-14 Available from: http://www.cdc.gov/ mmwr/preview/mmwrhtml/00001769.htm (Accessed 12 July 2016).

8. Dean AG, Sullivan KM, Soe MM. OpenEpi: Open Source Epidemiologic Statistics for Public Health, Version. www.OpenEpi.com updated 2015/05/04, (Accessed 19 May 2016) Available from: http://http://www.openepi.com/Menu/ OE_Menu.htm.

9. Section 1: General Principles and Practices for Disease Surveillance. Available from: http://www.idsp.nic.in/.../DistrictOpsMan/OManualDSU.pdf (Accessed 12 July 2016)
10. Mplementation Completion And Results: Report To The Republic of India for Integrated Disease Surveillance Project. Document of World Bank. Available from: http://www.idsp.nic.in/ (Accessed 21 August 2016).

11. Bagonza J, Kibira SPS, Rutebemberwa E. Performance of community health workers managing malaria, pneumonia and diarrhoea under the community case management programme in central Uganda: a cross sectional study. Malar J. 2014;13(1)367 Available from: http://www.malariajournal.com/ content/13/1/367.

12. Traoré M, Arsenault C, Schoemaker-Marcotte $C$, et al. Obstetric competence among primary healthcare workers in Mali. Int J Gynecol Obstet. 2014;126(1) 50-55 Available from: http://linkinghub.elsevier.com/retrieve/pii/ S0020729214001702.

13. Reijn E, Swaan CM, Kretzschmar MEE. Steenbergen JE Van: Analysis of timeliness of infectious disease reporting in the Netherlands. BMC Public Health. 2011;11:409.

14. Jajosky RA, Groseclose SL. Evaluation of reporting timeliness of public health surveillance systems for infectious diseases. BMC Public Health. 2004;4:29.

15. Reintjes $R$, Thelen $M$, Reiche $R$, et al. Benchmarking national surveillance systems: a new tool for the comparison of communicable disease surveillance and control in Europe. Eur. J. Public Health. 2006;17(4)375-380 Available from: http://eurpub.oxfordjournals.org/cgi/content/full/17/4/375. 